\title{
PHRYNICHOS BEIM GEWÄHRSMANN UND BEIM ABSCHREIBER AMMIANS
}

\author{
F. X. Ryan
}

\begin{abstract}
The article examines and finds wanting a recent thesis according to which a source used by Ammianus Marcellinus knew that Phrynichos produced his play on the sack of Miletos only after Athens had been sacked; then it proceeds to ask whether the chronological information vainly sought in the source of Ammianus has been preserved from another source by a copyist of Ammianus.
\end{abstract}

Vor kurzem stellte E. Badian fest, Ammians Gewährsmann habe gewußt, daß Phrynichos seine Einnahme Milets erst nach der Zerstörung Athens auf die Bühne brachte. ${ }^{1}$ Vorher hatte J. Roisman dem Wortlaut des herodoteischen Berichtes (Hdt. 6.21.2) entnommen, daß Herodot selbst von der Spätdatierung des Stückes ausgegangen sei: "das eigene Unglück," an das Phrynichos die Athener erinnert haben soll, sei die Verwüstung Athens gewesen. ${ }^{2}$ Auch Badian war der Meinung, daß Herodot wie er das Stück auf die siebziger Jahre setzte. ${ }^{3}$ Badian und Roisman haben jedoch dabei stillschweigend angenommen, daß Herodot den Ausdruck richtig verstand, und glaubten, die herodoteische Auffassung des Ausdruckes ermittelt zu haben, indem sie die richtige Auffassung desselben ermittelten. ${ }^{4}$ Man kann sich aber des Eindrucks nicht erwehren, daß Herodot selbst, wie Ammian auch, von der Aufführung der Tragödie bald nach der Erstürmung Milets ausging: schließlich haben sich einige der Historiker, die das Stück in bzw. um das Jahr 493/2 datieren, allein auf ihn berufen. Der Gewährsmann Ammians wäre dann der einzige, noch faßbare Schriftsteller, der den Vorfall richtigerweise auf die siebziger Jahre setzte. Wir bestreiten nicht, daß der Wortlaut des herodoteischen Berichtes die Herabdatierung des Aufführungsjahres zuläßt, sondern sind sogar davon überzeugt, daß die Herabdatierung - entgegen der in der Forschung bis in die letzte Zeit hinein einhellig vertretenen Ansicht und, wie wir glauben, entgegen der Ansicht Herodots auch-richtig ist. Es geht uns also im Folgenden nicht um das eigentliche Aufführungsjahr, auch nicht um das Aufführungsjahr bei Ammian, sondern zunächst um das Aufführungsjahr beim Gewährsmann Ammians, wenn denn überhaupt eines zu ermitteln ist.

Als es ihm zufiel, über Grausamkeiten zu berichten, vertraute Ammian auf praesentis temporis modestia (Amm. 28.1.2), anstatt mit der schlechten Behandlung, die apud vete-

\footnotetext{
${ }^{1}$ Badian 1996: 60.

2 Vgl. Roisman 1988: 18.

${ }^{3}$ Badian 1996: 59.

${ }^{4}$ Auch wenn man den Prozeß gegen Phrynichos recht spät (ca. 475), die schriftstellerische Tätigkeit Herodots ihrerseits sehr früh (ca. 450) ansetzt, ist das Intervall zwischen dem Ereignis und dem schriftlichen Bericht darüber groß genug, um Mißverständnisse aufkommen zu lassen.
} 
res vorkam, zu rechnen. Dann führte er ein Beispiel an für das, was ihm nicht widerfahren würde. ${ }^{5}$

hoc argumentum ${ }^{6}$ paulo postea digestum tumore tragico Phrynichus in theatrum induxerat Athenarum paulisperque iucunde auditus, cum coturnatius stilus procederet lacrimosus, indignatione damnatus est populi arbitrati non consolandi gratia, sed probrose monendi, quae pertulerat amabilis civitas nullis auctorum adminiculis fulta, hos quoque dolores scaenicis adnumerasse fabulis insolenter. erat enim Atheniensium colonia Miletus deducta inter Ionas alios per Nileum filium Codri, qui fertur pro patria bello se Dorico devovisse (Amm. 28.1.4).

Phrynichos versuchte, so Badian, "to 'console' the Athenians for the recent destruction of their city by showing that others had suffered a worse fate. The words consolandi gratia, describing the intention that misfired, show that the source (though obviously not Ammianus) was well aware of the fact that the play was produced after, indeed soon after, Xerxes' war."7 Aber die Worte consolandi gratia "show that the source...was well aware of the fact that the play was produced after...Xerxes' war" nur dann, wenn man consolandi gratia so definiert, wie Badian es tat. Wenn consolandi gratia heißen müßte, "to 'console' the Athenians for the recent destruction of their city by showing that others had suffered a worse fate," dann wäre es möglich, das Gut des Gewährsmannes von der eigenen Zutat Ammians zu trennen. Daß es aber nicht nötig ist, die fraglichen Worte so zu verstehen, zeigt schon der Umstand, daß Ammian selbst den Ausdruck anders auffaßte. Offenbar glaubte Ammian, daß Phrynichos gesucht hätte, den Athenern bei der Bewältigung des Unglücks der Milesier zu helfen. Der Ausdruck consolandi gratia liefert also keinen Beweis, daß Ammians Gewährsmann besser unterrichtet war als er selbst.

Die Suche nach einem Beleg für die Spätdatierung der Aufführung der Tragödie ist damit aber noch nicht erledigt. Ein solcher Beleg findet sich tatsächlich bei Ammian. Er begegnet uns indes nicht in den neueren Ausgaben, ${ }^{8}$ sondern in einer der Handschriften. Im Codex E stehen nach insolenter drei weitere Worte: Miletum relegatus est. ${ }^{9}$ Seyfarth machte darauf aufmerksam, daß die Erklärung erat enim Atheniensium colonia Miletus durch diesen Einschub einen anderen Sinn bekomme: In den Ausgaben mache die Erwähnung der colonia klar, warum die Zuschauermenge des Trostes be-

\footnotetext{
${ }^{5}$ Ammian wollte also seine Belesenheit unter Beweis stellen. Aus seiner Sicht wurde die Geschichte des Phrynichos nicht “at disproportionate length" (so aber Thompson 1947: 101) erzählt; tatsächlich verspricht er, sich kurz zu fassen (Amm. 28.1.2: docere succincte). Was W. Seyfarth (1978: 324 A. 5) in seinen Erläuterungen schreibt-“Ammian möchte mit der Anführung dieser Episode dem Vorwurf begegnen, er wolle die nachfolgenden Berichte zum Schaden der Stadt Rom an den Pranger stellen" - scheint das Richtige nicht getroffen zu haben. Ein ähnlich lautender Vorwurf gegen Phrynichos findet sich zwar bei Ammian, aber der athenische Tragödiendichter wurde ja nicht freigesprochen, sondern verurteilt. Die Moral der Geschichte hieß also nicht, wer Grausamkeiten schildere, der werde trotzdem glimpflich davonkommen.

${ }^{6}$ Zur Übersetzung s. Sabbah (1978: 386): “argumentum désigne le sujet d'une pièce de théâtre.”

${ }^{7}$ Badian 1996: 60.

${ }^{8}$ Der Beleg wurde jedoch in den Text der i. J. 1533 erschienenen Ausgaben des Gelenius und des Accursius aufgenommen; dazu s. Seyfarth 1962: 64-65.

9 Sie stehen im Apparat bei C. U. Clark (Berlin: Weidmann, 1915) und bei M.-A. Marié (Paris: Budé, 1984), mitunter fehlen sie aber dort, wie bei J. C. Rolfe (Cambridge, Mass.: Loeb, 1939) und sogar bei Seyfarth (1978) selbst.
} 
durfte, aber die erklärenden Worte "dienen im Codex E zur Begründung dessen, daß Phrynichos angeblich nach Milet verbannt wurde." 10 Seyfarth glaubte nicht, daß der Einschub in einer älteren, von MV unabhängigen Ammianhandschrift gestanden hätte, auch nicht, daß der Schreiber des Codex E "irgendeinen Anhalt" für den Einschub gehabt habe. Für wahrscheinlich hielt Seyfarth, daß die auf das Verjagen aus dem Theater zu beziehende Angabe, die Athener $\dot{\varepsilon} \xi \hat{\varepsilon} \beta \alpha \lambda$ ov den Dichter (Ail. VH 13.17, Schol. Ar. Vesp. 1490), den Schreiber des Codex E an eine Verbannung zu denken veranlasste: "Der Schreiber E vermißte an der umstrittenen Stelle eine nähere Bezeichnung der von Ammianus Marcellinus erwähnten Strafe und interpolierte die Worte Miletum relegatus est, wobei ihm vielleicht die mißverstandene Aelian-Stelle erinnerlich wurde." ${ }^{11}$

Der Umstand, daß Ammian nicht näher auf die Strafe einging, erklärt sich möglicherweise dadurch, daß er bei der Abfassung seines Werkes spätere Quellen benutzte, die die Strafe mit keinem Wort (wie Ps.-Longin. Subl. 24.1) oder allenfalls die Verhängung einer Geldstrafe erwähnten, ohne aber deren Höhe anzugeben (wie Plut. Praec. ger. reip. 17 [Mor. 814B]). ${ }^{12}$ In unserem Zusammenhang stellt sich die Frage, ob der Schreiber des Codex E aus anderer Quelle wußte, ${ }^{13}$ daß die "schwülstige Tragödie"14 erst in den siebziger Jahren aufgeführt wurde. Eine Verbannung nach Milet wird nämlich erst nach dem Sieg von Mykale denkbar, denn dieser Sieg "befreite Ionien von der Fremdherrschaft,"15 und davor lag Milet noch in Schutt und Asche. Beim völligen Schweigen der sonstigen Überlieferung über die Verbannung nach Milet wird man die Nachricht natürlich nicht für historisch halten wollen, es geht uns hier also ausschließlich darum, ob die unhistorische Nachricht Kenntnis des Aufführungsjahres voraussetzt. Gerade der Umstand, daß der Schreiber des Codex E die Verbannung nach Milet erfand oder auch nur übernahm, zeigt jedoch, daß es um seinen historischen Verstand nicht bestens bestellt war. Ohne weiteres möglich erscheint für ihn folgender Gedankengang: Phrynichos sei athenerfeindlich und milesierfreundlich gewesen-Milet sei eine Kolonie Athens gewesen-Athen habe den Dichter nach Milet verbannt. Schließlich ist darum an dieser Stelle von einer Verbannung die Rede, weil der Schreiber des Codex E weder die von Herodot erwähnten Strafen ${ }^{16}$ kannte noch sich die Mühe gab, sie zu erfahren. ${ }^{17}$ Es ist dann kaum anzunehmen, daß er sich nach dem Aufführungsjahr erkündigte oder dasselbe be-

\footnotetext{
10 Seyfarth 1962: 63.

${ }^{11}$ Seyfarth 1962: 64.

${ }_{12}$ Wenn die Stellen der Deklamationen des Libanios, die zeigen, daß es zu einem Eklat kam (Liban. Decl. 13.54, 19.26), erst nach dessen Tode publiziert wurden, dann werden sie von Ammian nicht konsultiert worden sein.

${ }^{13}$ Bei Texteinschüben erhebt sich die Frage, "ob sie echte Interpolationen des Schreibers E sind oder ob sie von diesem von dritter Seite her übernommen sind und aus welcher Quelle sie gegebenenfalls stammen" (Seyfarth 1962: 62). Man muß natürlich auch mit einer weiteren Möglichkeit rechnen: "Schließlich könnten spätere Gelehrte derartige Nachträge im Codex E vorgenommen haben" (Seyfarth 1962: 9).

${ }^{14}$ So wurde tumore tragico von O. Veh (Artemis Verlag: Zürich 1974) übersetzt; vgl. Seyfarth 1978 [1971]:

"in einer schwülstigen Tragödie."

${ }^{15}$ Hiller v. Gaertringen 1932: 1598.

${ }^{16} \mathrm{Zu}$ ihnen und dem Verfahren s. Ryan 2005: 1-4.

${ }^{17}$ Der Schreiber E “konnte wohl griechisch lesen und schreiben, wie die von ihm griechisch geschriebenen Partien seines Codex erkennen lassen” (Seyfarth 1962: 64).
} 
rücksichtigte, als er sich ans Werk machte. Die fraglichen Worte sind also ein Einschiebsel des Abschreibers. ${ }^{18}$

Auch nicht für den hier in Rede stehenden Abschreiber Ammians darf man also feststellen, daß er dank einer ihm zugänglichen Quelle das Drama in die siebziger Jahre datierte. Mithin geht keine, noch faßbare antike Quelle von einer Aufführung nach der Verwüstung Athens aus. Wenn nun die uns nicht erhaltenen Werke in dem Maße von Herodot abhängig waren, wie die uns erhaltenen zu sein scheinen, dann hat wohl kein antiker Schriftsteller je erkannt, daß die Tragödie erst in den siebziger Jahren aufgeführt wurde. Mit anderen Worten: Die Arbeit, die der Forschung in der Spätdatierung des Stückes zuvorkam, ist wahrscheinlich nicht verloren gegangen, sondern sie hat wohl nie existiert.

Dr. Francis X. Ryan

Department of Classics, University of Cincinnati

E-mail:fxryan@gmx.de

\section{Bibliographie}

Badian, E. (1996) 'Phrynichos and Athens' oì

Hiller von Gaertringen, F. Frhr. (1932) 'Miletos (Geschichte).' - RE 15, 1586-1622.

Roisman, J. (1988) 'On Phrynichos' Sack of Miletos and Phoinissai.' - Eranos 86, 15-23.

Ryan, F. X. (2005) 'Phrynichos kommt vor Gericht.' - SHT 6.A.1, 1-5.

Sabbah, G. (1978) La méthode d'Ammien Marcellin. Recherches sur la construction du discours historique dans les Res gestae. Paris: Les Belles Lettres.

Seyfarth, W. (1962) Der Codex Fuldensis und der Codex E des Ammianus Marcellinus. Zur Frage der handschriftlichen Überlieferung des Werkes des letzten römischen Geschichtsschreibers. Berlin: Akademie-Verlag (Abh.d. Dt. Akad. d. Wiss., Kl.f. Sprachen, Literatur u. Kunst; Jg. 1962, Nr. 2.)

Seyfarth, W. (1978) Ammianus Marcellinus, Römische Geschichte. Lateinisch und Deutsch und mit einem Kommentar versehen. 2., berichtigte Aufl. [zuerst 1971]. Darmstadt: Wissenschaftliche Buchgesellschaft.

Thompson, E. A. (1947) The Historical Work of Ammianus Marcellinus. Cambridge: University Press.

\footnotetext{
${ }^{18}$ Vgl. Seyfarth (1962: 66), der bei seiner Untersuchung zum Ergebnis kam, “daß die einzige Vorlage des Codex E der Fuldensis war. Die Abweichungen des Codex E von seiner Vorlage, ob sie nun im Text selbst stehen oder in Randbemerkungen enthalten sind, haben keinerlei Wert als Überlieferung, sind vielmehr bewußte oder unbewußte Änderungen des Schreibers E."
} 